Acta Crystallographica Section E

Structure Reports

Online

ISSN 1600-5368

Kathrine V. Booth, ${ }^{a}$ David J. Watkin, ${ }^{b}$ Sarah F. Jenkinson ${ }^{a}$ and George W. J. Fleet ${ }^{a}$

${ }^{a}$ Department of Organic Chemistry, Chemistry Research Laboratory, University of Oxford, Mansfield Road, Oxford OX1 3TA, England, and

b Department of Chemical Chrystallography, Chemistry Research Laboratory, University of Oxford, Mansfield Road, Oxford OX1 3TA, England

Correspondence e-mail:

victoria.booth@chem.ox.ac.uk

\section{Key indicators}

Single-crystal X-ray study

$T=150 \mathrm{~K}$

Mean $\sigma(\mathrm{C}-\mathrm{C})=0.003 \AA$

$R$ factor $=0.038$

$w R$ factor $=0.102$

Data-to-parameter ratio $=10.4$

For details of how these key indicators were automatically derived from the article, see http://journals.iucr.org/e.

\title{
2,4-Dimethyl-3,4-O-isopropylidene-L-arabinono- 1,5-lactone
}

The relative configuration at $\mathrm{C}-2$ of 2,4-dimethyl-3,4- $O$ isopropylidene-L-arabinono lactone, $\mathrm{C}_{10} \mathrm{H}_{16} \mathrm{O}_{5}$, which exists in the boat form, was unequivocally established by X-ray crystallographic analysis. The absolute configuration was determined by the use of 2-C-methyl-D-ribonolactone as a starting material.

\section{Comment}

Rare and new monosaccharides have potential both as healthy dietary alternatives (Sun et al., 2007; Skytte, 2002) and for specific chemotherapeutic uses (Nakajima et al., 2004; Menavuvu et al., 2006; Hossain et al., 2006). Branched 2-C-methyl pentoses have become readily available by treatment of an Amadori ketose with aqueous calcium hydroxide (Hotchkiss et al., 2007) and are key intermediates in the synthesis of 2-Cmethyl nucleosides, a new class of drugs for the treatment of hepatitis C (Sorbera et al., 2006). Carbohydrates with a branch at C-2 may also be accessed by the reaction of ketoses and deoxyketoses with cyanide (Hotchkiss et al., 2004; Soengas et al., 2005). In contrast, there have been no biological studies on unprotected monosaccharides with more than one carbon branch.

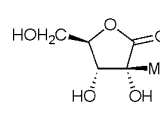

(1)

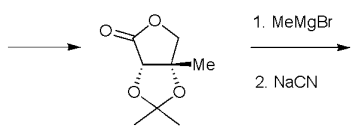

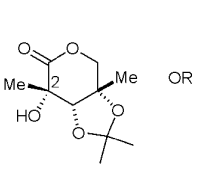

(3)
Received 26 January 2007
Accepted 29 January 2007

The protected lactone (4) is a key intermediate in the synthesis of monosaccharides with two $C$-methyl branches (Booth et al., 2007). 2-C-Methyl-D-ribonolactone, (1), prepared by the green environmentally friendly aqueous isomerization of D-glucose (Hotchkiss et al., 2006), may be converted to the 3-C-methyl-L-erythronolactone (2) as previously described (Barrett \& Dhanak, 1987; Barrett et al., 1989). Sequential treatment of (2) with methyl magnesium bromide followed by aqueous cyanide leads to the isolation of a major crystalline product which has a new stereogenic centre, which could be either the epimeric ribo- (3) or arabinolactone (4). X-ray crystallographic analysis resolved the ambiguity at $\mathrm{C}-2$ and unequivocally established the relative stereochemistry as the arabinono-1,5-lactone (4), which exists in a boat form; the absolute configuration of (4) is determined by the use of 2-C-methyl-D-ribonolactone (1) as the starting material.

The molecular structure of (4) is shown in Fig. 1. The molecular geometry contains no unusual features. The largest 


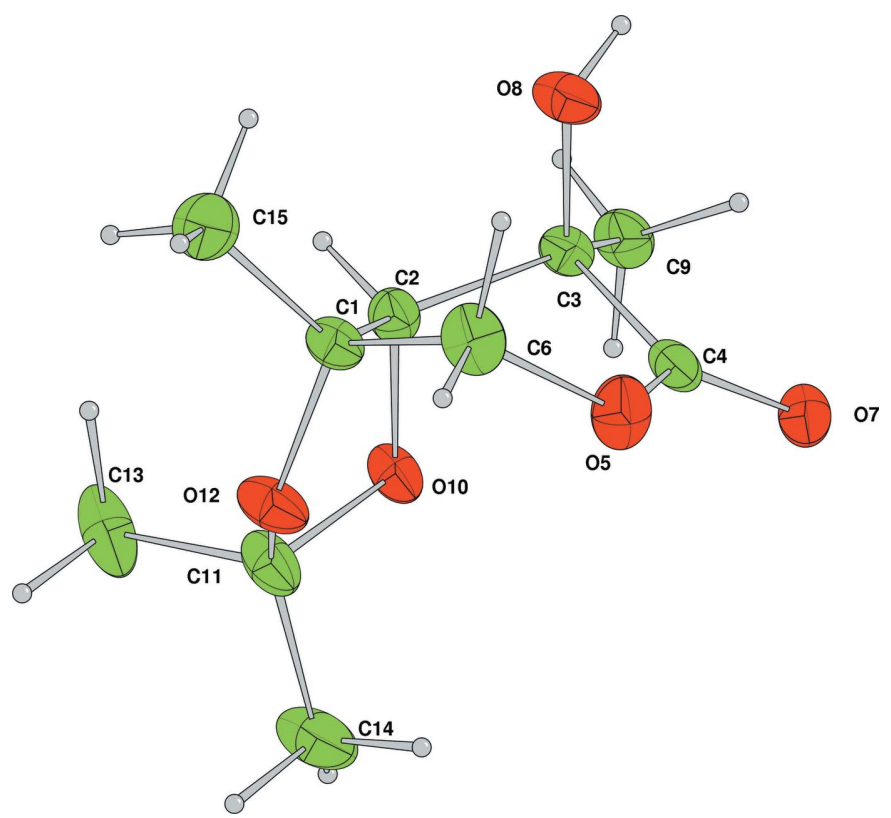

Figure 1

The molecular structure of the title compound with displacement ellipsoids drawn at the $50 \%$ probability level. $\mathrm{H}$ atoms are shown as spheres of arbitrary radius.

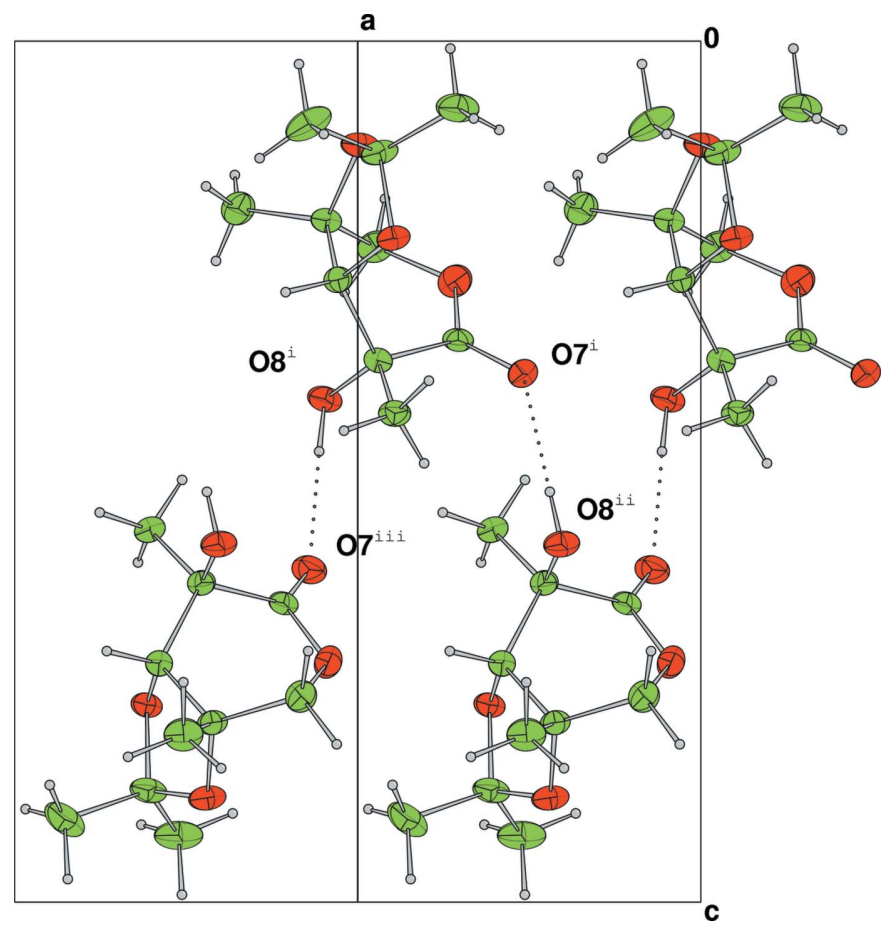

Figure 2

Part of the hydrogen-bonded (dotted lines) ribbon lying parallel to $a$. The image has been rotated about $c$ (out of the plane of the ribbon) to clarify the hydrogen-bonding chain. The molecule corresponding to the published coordinates does not form any hydrogen bonds within the natural unit cell. [Symmetry codes: (i) $-x+1, y-\frac{1}{2},-z+\frac{1}{2}$; (ii) $-x+\frac{1}{2}$, $-y+1, z+\frac{1}{2}$; (iii) $-x+\frac{3}{2},-y+1, z+\frac{1}{2}$. $]$

differences from the Mogul norms (Bruno et al., 2004) are C2-O3 (0.02 А, Mogul s.u. 0.01 А) and C3-C9-O8 (3.4 Mogul s.u. $\left.1.9^{\circ}\right)$. The crystal structure consists of broad ribbons of hydrogen-bonded molecules lying with the plane of the ribbon perpendicular to $b$, and the length of the ribbon lying along $a$ (Fig. 2). The hydrogen bonds form the backbone of the ribbon, with the individual molecules lying alternately on either side. The backbone of each ribbon lies above and parallel to the interface between two ribbons in the adjacent layers.

\section{Experimental}

2,4-Dimethyl-3,4-O-isopropylidene-L-arabinono lactone (4) was crystallized from a mix of ethyl acetate and cyclohexane by vapour diffusion: m.p. $385-391 \mathrm{~K} ;[\alpha]_{D}^{23}+131$ (c, 1.5 in chloroform)

\section{Crystal data}

$\mathrm{C}_{10} \mathrm{H}_{16} \mathrm{O}_{5}$

$M_{r}=216.23$

Orthorhombic, $P 2_{1} 2_{1} 2_{1}$

$a=6.3457$ (2) ^

$b=12.0530(4) \AA$

$c=14.1034(5) \AA$

$V=1078.69(6) \AA^{3}$

$$
\begin{aligned}
& Z=4 \\
& D_{x}=1.331 \mathrm{Mg} \mathrm{m}^{-3} \\
& \text { Mo } K \alpha \text { radiation } \\
& \mu=0.11 \mathrm{~mm}^{-1} \\
& T=150 \mathrm{~K} \\
& \text { Plate, colourless } \\
& 0.40 \times 0.40 \times 0.20 \mathrm{~mm}
\end{aligned}
$$

\section{Data collection}

Nonius KappaCCD diffractometer $\omega$ scans

Absorption correction: multi-scan

(DENZO/SCALEPACK;

Otwinowski \& Minor, 1997)

$T_{\text {min }}=0.82, T_{\text {max }}=0.98$

6458 measured reflections 1421 independent reflections 1301 reflections with $I>2 \sigma(I)$

$R_{\text {int }}=0.051$

$\theta_{\max }=27.5^{\circ}$

\section{Refinement}

Refinement on $F^{2}$

$R\left[F^{2}>2 \sigma\left(F^{2}\right)\right]=0.038$

$w R\left(F^{2}\right)=0.102$

$S=0.86$

1421 reflections

136 parameters

$\mathrm{H}$-atom parameters constrained

$$
\begin{aligned}
& w=1 /\left[\sigma^{2}\left(F^{2}\right)+(0.07 P)^{2}\right. \\
& \quad+0.5 P], \\
& \text { where } P=\left[\max \left(F_{\mathrm{o}}{ }^{2}, 0\right)+2 F_{\mathrm{c}}{ }^{2}\right] / 3 \\
& (\Delta / \sigma)_{\max }<0.001 \\
& \Delta \rho_{\max }=0.26 \mathrm{e} \AA^{-3} \\
& \Delta \rho_{\min }=-0.18 \mathrm{e} \AA^{-3}
\end{aligned}
$$

Table 1

Hydrogen-bond geometry $\left(\AA,{ }^{\circ}\right)$.

\begin{tabular}{lllll}
\hline$D-\mathrm{H} \cdots A$ & \multicolumn{1}{c}{$D-\mathrm{H}$} & $\mathrm{H} \cdots A$ & $D \cdots A$ & $D-\mathrm{H} \cdots A$ \\
\hline $\mathrm{O} 8-\mathrm{H} 1 \cdots \mathrm{O}^{\text {iv }}$ & 0.88 & 1.99 & $2.870(2)$ & 178 \\
\hline Symmetry code: (iv) $x-\frac{1}{2},-y+\frac{3}{2},-z$. &
\end{tabular}

In the absence of significant anomalous scattering, Friedel pairs were merged and the absolute configuration was assigned from the starting material. The relatively large ratio of minimum to maximum corrections applied in the multi-scan process (1:1.2) reflects effects in addition to absorption, possibly connected with the flake-like aspect of the sample. Changes in illuminated volume were kept to a minimum, and were taken into account (Görbitz, 1999) by the multiscan inter-frame scaling (DENZO/SCALEPACK; Otwinowski \& Minor, 1997).

The $\mathrm{H}$ atoms were all located in a difference map, but those attached to $\mathrm{C}$ atoms were repositioned geometrically. The $\mathrm{H}$ atoms were initially refined with soft restraints on the bond lengths and angles to regularize their geometry $(\mathrm{C}-\mathrm{H}$ in the range $0.93-0.98 \AA$, $\mathrm{O}-\mathrm{H}=0.82 \AA$ ) and $U_{\text {iso }}(\mathrm{H})$ (in the range 1.2-1.5 times $U_{\text {eq }}$ of the parent atom), after which the positions were refined with riding constraints. 
Data collection: COLLECT (Nonius, 2001); cell refinement: DENZO/SCALEPACK (Otwinowski \& Minor, 1997); data reduction: DENZO/SCALEPACK; program(s) used to solve structure: SIR92 (Altomare et al., 1994); program(s) used to refine structure: CRYSTALS (Betteridge et al., 2003); molecular graphics: CAMERON (Watkin et al., 1996); software used to prepare material for publication: CRYSTALS.

A generous gift of 2-C-methyl-D-ribonolactone from Novartis Pharma AG Basel is gratefully acknowledged.

\section{References}

Altomare, A., Cascarano, G., Giacovazzo, G., Guagliardi, A., Burla, M. C., Polidori, G. \& Camalli, M. (1994). J. Appl. Cryst. 27, 435.

Barrett, A. G. M., Bezuidenhoudt, B. C. B., Dhanak, D., Gasiecki, A. F., Howell, A. R., Lee, A. C. \& Russell, M. A. (1989). J. Org. Chem. 54, 33213324

Barrett, A. G. M. \& Dhanak, D. (1987). Tetrahedron Lett. 28, 3327-3330.

Betteridge, P. W., Carruthers, J. R., Cooper, R. I., Prout, K. \& Watkin, D. J. (2003). J. Appl. Cryst. 36, 1487.

Booth, V. K., Best, D., Jenkinson, S. F. \& Fleet, G. W. J. (2007). In preparation.

Bruno, I. J., Cole, J. C., Kessler, M., Luo, J., Motherwell, W. D. S., Purkis, L. H., Smith, B. R., Taylor, R., Cooper, R. I., Harris, S. E. \& Orpen, A. G. (2004). J. Chem. Inf. Comput. Sci. 44, 2133-2144.
Görbitz, C. H. (1999). Acta Cryst. B55, 1090-1098.

Hossain, M. A., Wakabayashi, H., Izuishi, K., Okano, K., Yachida, S., Tokuda, M., Izumori, K. \& Maeta, H. J. (2006). Biosci. Bioeng. 101, 369-371.

Hotchkiss, D. J., Jenkinson, S. F., Storer, R., Heinz, T. \& Fleet, G. W. J. (2006). Tetrahedron Lett. 47, 315-318.

Hotchkiss, D. J., Soengas, R., Booth, K. V., Weymouth-Wilson, A. C., EastwickField, V. \& Fleet, G. W. J. (2007). Tetrahedron Lett. 48, 517-520.

Hotchkiss, D. J., Soengas, R., Simone, M. I., van Ameijde, J., Hunter, S., Cowley, A. R. \& Fleet, G. W. J. (2004). Tetrahedron Lett. 45, 9461-9464.

Menavuvu, B. T., Poonperm, W., Leang, K., Noguchi, N., Okada, H., Morimoto, K., Granstrom, T. B., Takada, G. \& Izumori, K. J. (2006). Biosci. Bioeng. 101, 340-345.

Nakajima, Y., Gotanda, T., Uchimiya, H., Furukawa, T., Haraguchi, M., Ikeda, R., Sumizawa, T., Yoshida, H. \& Akiyama, S. (2004). Cancer Res. 64, 1794 1801.

Nonius (2001). COLLECT. Nonius BV, Delft, The Netherlands.

Otwinowski, Z. \& Minor, W. (1997). Methods in Enzymology, Vol. 276, Macromolecular Crystallography, Part A, edited by C. W. Carter Jr \& R. M. Sweet, pp. 307-326. New York: Academic Press.

Skytte, U. P. (2002). Cereal Foods World, 47, 224.

Soengas, R., Izumori, K., Simone, M. I., Watkin, D. J., Skytte, U. P., Soetaert, W. \& Fleet, G. W. J. (2005). Tetrahedron Lett. 46, 5755-5759.

Sorbera, L. A., Castaner, J. \& Leeson, P. A. (2006). Drugs Future, 31, 320 324.

Sun, Y. X., Hayakawa, S., Ogawa, M. \& Izumori, K. (2007). Food Contr. 18, 220-227.

Watkin, D. J., Prout, C. K. \& Pearce, L. J. (1996). CAMERON. Chemical Crystallography Laboratory, Oxford, England. 


\title{
supporting information
}

Acta Cryst. (2007). E63, o1128-o1130 [https://doi.org/10.1107/S160053680700476X]

\section{2,4-Dimethyl-3,4-O-isopropylidene-L-arabinono-1,5-lactone}

\author{
Kathrine V. Booth, David J. Watkin, Sarah F. Jenkinson and George W. J. Fleet
}

2,4-Dimethyl-3,4-O-isopropylidene- $L$-arabinono lactone

Crystal data

$\mathrm{C}_{10} \mathrm{H}_{16} \mathrm{O}_{5}$

$M_{r}=216.23$

Orthorhombic, $P 2_{1} 2_{1} 2_{1}$

Hall symbol: P $2 \mathrm{ac} 2 \mathrm{ab}$

$a=6.3457(2) \AA$

$b=12.0530(4) \AA$

$c=14.1034(5) \AA$

$V=1078.69(6) \AA^{3}$

$Z=4$

\section{Data collection}

Nonius KappaCCD

diffractometer

Graphite monochromator

$\omega$ scans

Absorption correction: multi-scan

(DENZO/SCALEPACK; Otwinowski \& Minor, 1997)

$T_{\min }=0.82, T_{\max }=0.98$

Refinement

Refinement on $F^{2}$

Least-squares matrix: full

$R\left[F^{2}>2 \sigma\left(F^{2}\right)\right]=0.038$

$w R\left(F^{2}\right)=0.102$

$S=0.86$

1421 reflections

136 parameters

0 restraints
$F(000)=464$

$D_{\mathrm{x}}=1.331 \mathrm{Mg} \mathrm{m}^{-3}$

Mo $K \alpha$ radiation, $\lambda=0.71073 \AA$

Cell parameters from 1339 reflections

$\theta=5-27^{\circ}$

$\mu=0.11 \mathrm{~mm}^{-1}$

$T=150 \mathrm{~K}$

Plate, colourless

$0.40 \times 0.40 \times 0.20 \mathrm{~mm}$

6458 measured reflections

1421 independent reflections

1301 reflections with $I>2 \sigma(I)$

$R_{\text {int }}=0.051$

$\theta_{\max }=27.5^{\circ}, \theta_{\min }=5.3^{\circ}$

$h=-8 \rightarrow 8$

$k=-15 \rightarrow 15$

$l=-18 \rightarrow 18$

Primary atom site location: structure-invariant direct methods

Hydrogen site location: inferred from neighbouring sites

$\mathrm{H}$-atom parameters constrained

$w=1 /\left[\sigma^{2}\left(F^{2}\right)+(0.07 P)^{2}+0.5 P\right]$,

where $P=\left[\max \left(F_{\mathrm{o}}{ }^{2}, 0\right)+2 F_{\mathrm{c}}{ }^{2}\right] / 3$

$(\Delta / \sigma)_{\max }=0.000299$

$\Delta \rho_{\max }=0.26 \mathrm{e} \AA^{-3}$

$\Delta \rho_{\min }=-0.18$ e $\AA^{-3}$

Fractional atomic coordinates and isotropic or equivalent isotropic displacement parameters $\left(\AA^{2}\right)$

\begin{tabular}{lllll}
\hline & $x$ & $y$ & $z$ & $U_{\text {iso }} * / U_{\text {eq }}$ \\
\hline C1 & $0.2418(3)$ & $0.83400(16)$ & $0.29155(13)$ & 0.0226 \\
C2 & $0.1810(3)$ & $0.73928(16)$ & $0.22279(13)$ & 0.0215 \\
C3 & $0.3015(3)$ & $0.74232(16)$ & $0.12915(13)$ & 0.0209 \\
C4 & $0.5380(3)$ & $0.74540(17)$ & $0.15287(14)$ & 0.0234
\end{tabular}




$\begin{array}{lllll}\text { O5 } & 0.5983(2) & 0.81516(14) & 0.22116(11) & 0.0313 \\ \text { C6 } & 0.4429(4) & 0.89114(18) & 0.26103(16) & 0.0299 \\ \text { O7 } & 0.6698(2) & 0.68862(15) & 0.11399(10) & 0.0312 \\ \text { O8 } & 0.2491(3) & 0.84518(11) & 0.08425(10) & 0.0283 \\ \text { C9 } & 0.2504(4) & 0.64316(17) & 0.06758(14) & 0.0253 \\ \text { O10 } & 0.2439(2) & 0.64042(11) & 0.27115(9) & 0.0235 \\ \text { C11 } & 0.2262(4) & 0.66333(16) & 0.37041(14) & 0.0279 \\ \text { O12 } & 0.2837(3) & 0.77729(11) & 0.37897(9) & 0.0289 \\ \text { C13 } & 0.0003(5) & 0.6445(2) & 0.40394(18) & 0.0429 \\ \text { C14 } & 0.3840(5) & 0.5936(2) & 0.42241(15) & 0.0407 \\ \text { C15 } & 0.0713(4) & 0.92003(18) & 0.30548(17) & 0.0333 \\ \text { H21 } & 0.0287 & 0.7394 & 0.2085 & 0.0263^{*} \\ \text { H61 } & 0.5088 & 0.9295 & 0.3166 & 0.0383^{*} \\ \text { H62 } & 0.4079 & 0.9467 & 0.2084 & 0.0377^{*} \\ \text { H91 } & 0.3402 & 0.6474 & 0.0096 & 0.0402^{*} \\ \text { H92 } & 0.1033 & 0.6442 & 0.0475 & 0.0414^{*} \\ \text { H93 } & 0.2831 & 0.5762 & 0.1055 & 0.0416^{*} \\ \text { H131 } & -0.0088 & 0.6629 & 0.4733 & 0.0662^{*} \\ \text { H132 } & -0.0928 & 0.6940 & 0.3641 & 0.0656^{*} \\ \text { H133 } & -0.0343 & 0.5655 & 0.3920 & 0.0667^{*} \\ \text { H141 } & 0.3784 & 0.6088 & 0.4914 & 0.0584^{*} \\ \text { H142 } & 0.5234 & 0.6119 & 0.3969 & 0.0597^{*} \\ \text { H143 } & 0.3535 & 0.5157 & 0.4100 & 0.0591^{*} \\ \text { H151 } & 0.1210 & 0.9798 & 0.3445 & 0.0480^{*} \\ \text { H152 } & 0.0386 & 0.9522 & 0.2446 & 0.0501^{*} \\ \text { H153 } & -0.0564 & 0.8859 & 0.3313 & 0.0484^{*} \\ \text { H1 } & 0.2235 & 0.8363 & 0.0234 & 0.0405^{*}\end{array}$

Atomic displacement parameters $\left(\AA^{2}\right)$

\begin{tabular}{lllllll}
\hline & $U^{11}$ & $U^{22}$ & $U^{33}$ & $U^{12}$ & $U^{13}$ & $U^{23}$ \\
\hline C1 & $0.0272(10)$ & $0.0237(9)$ & $0.0169(8)$ & $-0.0009(9)$ & $-0.0020(8)$ & $-0.0002(7)$ \\
C2 & $0.0211(9)$ & $0.0243(9)$ & $0.0189(9)$ & $-0.0011(8)$ & $0.0007(7)$ & $-0.0011(7)$ \\
C3 & $0.0226(9)$ & $0.0223(9)$ & $0.0176(8)$ & $-0.0006(8)$ & $-0.0016(7)$ & $0.0009(7)$ \\
C4 & $0.0242(9)$ & $0.0309(10)$ & $0.0152(8)$ & $-0.0017(9)$ & $0.0010(8)$ & $0.0048(8)$ \\
O5 & $0.0234(7)$ & $0.0404(8)$ & $0.0301(8)$ & $-0.0062(7)$ & $-0.0002(7)$ & $-0.0081(7)$ \\
C6 & $0.0332(12)$ & $0.0278(10)$ & $0.0287(11)$ & $-0.0059(9)$ & $0.0009(10)$ & $-0.0062(9)$ \\
O7 & $0.0249(7)$ & $0.0461(9)$ & $0.0226(7)$ & $0.0056(7)$ & $0.0034(6)$ & $0.0007(7)$ \\
O8 & $0.0378(9)$ & $0.0276(7)$ & $0.0195(7)$ & $0.0021(7)$ & $-0.0042(6)$ & $0.0031(5)$ \\
C9 & $0.0280(10)$ & $0.0290(9)$ & $0.0190(8)$ & $-0.0017(10)$ & $-0.0025(8)$ & $-0.0035(7)$ \\
O10 & $0.0317(8)$ & $0.0217(6)$ & $0.0171(6)$ & $-0.0023(7)$ & $0.0037(6)$ & $-0.0005(5)$ \\
C11 & $0.0430(12)$ & $0.0232(9)$ & $0.0176(9)$ & $-0.0003(10)$ & $0.0063(9)$ & $-0.0023(7)$ \\
O12 & $0.0458(9)$ & $0.0237(7)$ & $0.0172(7)$ & $-0.0011(7)$ & $-0.0037(7)$ & $-0.0004(5)$ \\
C13 & $0.0550(16)$ & $0.0391(13)$ & $0.0345(13)$ & $-0.0082(12)$ & $0.0226(12)$ & $-0.0031(11)$ \\
C14 & $0.0671(18)$ & $0.0324(11)$ & $0.0226(10)$ & $0.0087(12)$ & $-0.0016(11)$ & $0.0031(9)$ \\
C15 & $0.0399(13)$ & $0.0294(10)$ & $0.0307(11)$ & $0.0070(10)$ & $0.0012(10)$ & $-0.0042(9)$ \\
& & & & & &
\end{tabular}


Geometric parameters $\left(\AA,{ }^{\circ}\right)$

\begin{tabular}{|c|c|c|c|}
\hline $\mathrm{C} 1-\mathrm{C} 2$ & $1.547(3)$ & C9-H91 & 0.998 \\
\hline $\mathrm{C} 1-\mathrm{C} 6$ & $1.513(3)$ & C9-H92 & 0.975 \\
\hline $\mathrm{C} 1-\mathrm{O} 12$ & $1.435(2)$ & $\mathrm{C} 9-\mathrm{H} 93$ & 0.990 \\
\hline $\mathrm{C} 1-\mathrm{C} 15$ & $1.511(3)$ & $\mathrm{O} 10-\mathrm{C} 11$ & $1.431(2)$ \\
\hline $\mathrm{C} 2-\mathrm{C} 3$ & $1.526(3)$ & $\mathrm{C} 11-\mathrm{O} 12$ & $1.426(2)$ \\
\hline $\mathrm{C} 2-\mathrm{O} 10$ & $1.430(2)$ & $\mathrm{C} 11-\mathrm{C} 13$ & $1.526(3)$ \\
\hline $\mathrm{C} 2-\mathrm{H} 21$ & 0.987 & $\mathrm{C} 11-\mathrm{C} 14$ & $1.499(3)$ \\
\hline $\mathrm{C} 3-\mathrm{C} 4$ & $1.538(3)$ & $\mathrm{C} 13-\mathrm{H} 131$ & 1.005 \\
\hline $\mathrm{C} 3-\mathrm{O} 8$ & $1.431(2)$ & $\mathrm{C} 13-\mathrm{H} 132$ & 1.010 \\
\hline $\mathrm{C} 3-\mathrm{C} 9$ & $1.512(3)$ & $\mathrm{C} 13-\mathrm{H} 133$ & 0.992 \\
\hline $\mathrm{C} 4-\mathrm{O} 5$ & $1.335(3)$ & $\mathrm{C} 14-\mathrm{H} 141$ & 0.991 \\
\hline $\mathrm{C} 4-\mathrm{O} 7$ & $1.212(3)$ & $\mathrm{C} 14-\mathrm{H} 142$ & 0.980 \\
\hline $\mathrm{O} 5-\mathrm{C} 6$ & $1.459(3)$ & $\mathrm{C} 14-\mathrm{H} 143$ & 0.974 \\
\hline C6-H61 & 1.002 & C15-H151 & 0.960 \\
\hline C6-H62 & 1.025 & $\mathrm{C} 15-\mathrm{H} 152$ & 0.965 \\
\hline $\mathrm{O} 8-\mathrm{H} 1$ & 0.880 & $\mathrm{C} 15-\mathrm{H} 153$ & 0.979 \\
\hline $\mathrm{C} 2-\mathrm{C} 1-\mathrm{C} 6$ & $111.59(16)$ & $\mathrm{H} 91-\mathrm{C} 9-\mathrm{H} 92$ & 107.9 \\
\hline $\mathrm{C} 2-\mathrm{C} 1-\mathrm{O} 12$ & $103.50(14)$ & $\mathrm{C} 3-\mathrm{C} 9-\mathrm{H} 93$ & 106.8 \\
\hline $\mathrm{C} 6-\mathrm{C} 1-\mathrm{O} 12$ & $107.74(17)$ & $\mathrm{H} 91-\mathrm{C} 9-\mathrm{H} 93$ & 111.4 \\
\hline $\mathrm{C} 2-\mathrm{C} 1-\mathrm{C} 15$ & $114.17(18)$ & $\mathrm{H} 92-\mathrm{C} 9-\mathrm{H} 93$ & 111.6 \\
\hline $\mathrm{C} 6-\mathrm{C} 1-\mathrm{C} 15$ & $109.17(17)$ & $\mathrm{C} 2-\mathrm{O} 10-\mathrm{C} 11$ & $106.50(14)$ \\
\hline $\mathrm{O} 12-\mathrm{C} 1-\mathrm{C} 15$ & $110.37(16)$ & $\mathrm{O} 10-\mathrm{C} 11-\mathrm{O} 12$ & $104.39(15)$ \\
\hline $\mathrm{C} 1-\mathrm{C} 2-\mathrm{C} 3$ & $113.57(16)$ & $\mathrm{O} 10-\mathrm{C} 11-\mathrm{C} 13$ & $110.36(19)$ \\
\hline $\mathrm{C} 1-\mathrm{C} 2-\mathrm{O} 10$ & $104.26(14)$ & $\mathrm{O} 12-\mathrm{C} 11-\mathrm{C} 13$ & $110.94(19)$ \\
\hline $\mathrm{C} 3-\mathrm{C} 2-\mathrm{O} 10$ & $107.03(15)$ & $\mathrm{O} 10-\mathrm{C} 11-\mathrm{C} 14$ & $108.53(17)$ \\
\hline $\mathrm{C} 1-\mathrm{C} 2-\mathrm{H} 21$ & 111.8 & $\mathrm{O} 12-\mathrm{C} 11-\mathrm{C} 14$ & $109.1(2)$ \\
\hline $\mathrm{C} 3-\mathrm{C} 2-\mathrm{H} 21$ & 108.3 & $\mathrm{C} 13-\mathrm{C} 11-\mathrm{C} 14$ & $113.12(19)$ \\
\hline $\mathrm{O} 10-\mathrm{C} 2-\mathrm{H} 21$ & 111.9 & $\mathrm{C} 1-\mathrm{O} 12-\mathrm{C} 11$ & $109.79(15)$ \\
\hline $\mathrm{C} 2-\mathrm{C} 3-\mathrm{C} 4$ & $107.53(16)$ & $\mathrm{C} 11-\mathrm{C} 13-\mathrm{H} 131$ & 108.8 \\
\hline $\mathrm{C} 2-\mathrm{C} 3-\mathrm{O} 8$ & $106.69(15)$ & $\mathrm{C} 11-\mathrm{C} 13-\mathrm{H} 132$ & 106.8 \\
\hline $\mathrm{C} 4-\mathrm{C} 3-\mathrm{O} 8$ & $107.58(16)$ & $\mathrm{H} 131-\mathrm{C} 13-\mathrm{H} 132$ & 112.2 \\
\hline $\mathrm{C} 2-\mathrm{C} 3-\mathrm{C} 9$ & $111.74(16)$ & C11-C13-H133 & 107.3 \\
\hline $\mathrm{C} 4-\mathrm{C} 3-\mathrm{C} 9$ & $110.67(17)$ & $\mathrm{H} 131-\mathrm{C} 13-\mathrm{H} 133$ & 111.4 \\
\hline $\mathrm{O} 8-\mathrm{C} 3-\mathrm{C} 9$ & $112.38(15)$ & $\mathrm{H} 132-\mathrm{C} 13-\mathrm{H} 133$ & 110.1 \\
\hline $\mathrm{C} 3-\mathrm{C} 4-\mathrm{O} 5$ & $116.88(18)$ & $\mathrm{C} 11-\mathrm{C} 14-\mathrm{H} 141$ & 110.7 \\
\hline $\mathrm{C} 3-\mathrm{C} 4-\mathrm{O} 7$ & $124.15(19)$ & C11-C14-H142 & 107.3 \\
\hline $\mathrm{O} 5-\mathrm{C} 4-\mathrm{O} 7$ & $118.97(19)$ & $\mathrm{H} 141-\mathrm{C} 14-\mathrm{H} 142$ & 110.6 \\
\hline $\mathrm{C} 4-\mathrm{O} 5-\mathrm{C} 6$ & $118.67(16)$ & $\mathrm{C} 11-\mathrm{C} 14-\mathrm{H} 143$ & 108.6 \\
\hline $\mathrm{C} 1-\mathrm{C} 6-\mathrm{O} 5$ & $113.22(16)$ & $\mathrm{H} 141-\mathrm{C} 14-\mathrm{H} 143$ & 110.3 \\
\hline $\mathrm{C} 1-\mathrm{C} 6-\mathrm{H} 61$ & 109.9 & $\mathrm{H} 142-\mathrm{C} 14-\mathrm{H} 143$ & 109.3 \\
\hline $\mathrm{O} 5-\mathrm{C} 6-\mathrm{H} 61$ & 108.0 & $\mathrm{C} 1-\mathrm{C} 15-\mathrm{H} 151$ & 110.7 \\
\hline $\mathrm{C} 1-\mathrm{C} 6-\mathrm{H} 62$ & 108.7 & $\mathrm{C} 1-\mathrm{C} 15-\mathrm{H} 152$ & 108.3 \\
\hline $\mathrm{O} 5-\mathrm{C} 6-\mathrm{H} 62$ & 106.1 & $\mathrm{H} 151-\mathrm{C} 15-\mathrm{H} 152$ & 106.3 \\
\hline $\mathrm{H} 61-\mathrm{C} 6-\mathrm{H} 62$ & 110.9 & $\mathrm{C} 1-\mathrm{C} 15-\mathrm{H} 153$ & 110.7 \\
\hline $\mathrm{C} 3-\mathrm{O} 8-\mathrm{H} 1$ & 111.6 & $\mathrm{H} 151-\mathrm{C} 15-\mathrm{H} 153$ & 111.9 \\
\hline
\end{tabular}


supporting information

$\begin{array}{llll}\mathrm{C} 3-\mathrm{C} 9-\mathrm{H} 91 & 107.9 & \mathrm{H} 152-\mathrm{C} 15-\mathrm{H} 153 & 108.8\end{array}$

$\mathrm{C} 3-\mathrm{C} 9-\mathrm{H} 92 \quad 111.2$

Hydrogen-bond geometry $\left(A,{ }^{\circ}\right)$

\begin{tabular}{lllll}
\hline$D-\mathrm{H} \cdots A$ & $D-\mathrm{H}$ & $\mathrm{H} \cdots A$ & $D \cdots A$ & $D-\mathrm{H} \cdots A$ \\
\hline $\mathrm{O} 8-\mathrm{H} 1 \cdots \mathrm{O} 7^{\mathrm{i}}$ & 0.88 & 1.99 & $2.870(2)$ & 178 \\
\hline
\end{tabular}

Symmetry code: (i) $x-1 / 2,-y+3 / 2,-z$. 